\title{
Subependymoma in the Lateral Ventricle Incidentally Detected by Routine Brain Examination
}

\author{
-Case Report-
}

\author{
Shigenori Katayama, Katsuzo Fujta, Naoya TAKeda, Kimio Hashimoto*, \\ Norihiko TAMAKI ${ }^{* *}$, and Masahiro ASADA***
}

Departments of Neurosurgery and *Pathology, Nishi-Kobe Medical Center, Kobe; ** Department of Neurosurgery, Kobe University School of Medicine, Kobe; ${ }^{* * *}$ Department of Neurosurgery,

Yodogawa Christian Hospital, Osaka

\begin{abstract}
A 42-year-old male visited our hospital for a routine brain examination, which incidentally identified an intraventricular mass lesion $\left(2.7 \times 1.6 \times 1.2 \mathrm{~cm}^{3}\right)$. Magnetic resonance imaging showed the tumor was isointense on the $T_{1}$-weighted image and hyperintense on the $T_{2}$-weighted and proton images. The intraventricular tumor was totally extirpated through the interhemispheric ipsilateral transcallosal approach. The histological diagnosis was subependymoma. Neuroimaging cannot differentiate this benign neoplasm from other more aggressive tumors. Widespread use of the medical checkup system is expected to find a higher incidence of otherwise non-identified asymptomatic lesions. Surgical extirpation is one of the treatment options to establish the correct diagnosis and to prevent symptoms.
\end{abstract}

Key words: subependymoma, intraventricular tumor, medical checkup

\section{Introduction}

Subependymoma is a rare, benign tumor of the central nervous system, first identified in $1945^{10)}$ and predominantly occurring in the ventricular system. Seven subependymomas $\{0.7 \%)$ were identified among 1000 intracranial neoplasms. ${ }^{\text {B) }}$ Most subependymomas are located in the fourth and the lateral ventricles. ${ }^{11,13)}$ Symptomatic subependymomas are sometimes identified due to hydrocephalus or the location-related symptoms. ${ }^{11)}$ Some subependymomas are asymptomatic and are only identified at postmortem examination. ${ }^{3,6,11)}$ Most surgically treated subependymomas have been symptomatic, and asymptomatic subependymomas have rarely been treated. We present a case of asymptomatic subependymoma incidentally identified during a routine medical examination and treated surgically.

\section{Case Report}

An asymptomatic 42-year-old male visited our

Received July 15, 1997; Accepted November 6, 1997 hospital for a routine brain examination, which incidentally identified an intraventricular mass lesion. Initial neurological examination was unremarkable. There was no family history of phakomatoses. Computed tomography (CT) demonstrated an oval, solid, isodense lesion in the right lateral ventricle without hydrocephalus. Although no apparent cystic component was observed, spotty low dense areas were identified inside the tumor. No calcification or subependymal nodule was present (Fig. 1). Magnetic resonance (MR) imaging depicted the lesion as isointense on the $\mathrm{T}_{1}$-weighted image, and hyperintense on the $\mathrm{T}_{2}$-weighted and proton images. The tumor was partially enhanced by gadoliniumdiethylenetriaminepenta-acetic acid (Gd-DTPA). The tumor, $2.7 \times 1.6 \times 1.2 \mathrm{~cm}^{3}$ in size, was located in the right lateral ventricular floor and compressed the septum pellucidum. No intraparenchymal extension was observed (Fig. 2). Cerebral angiography was unremarkable.

Right frontoparietal craniotomy was performed and the tumor was totally resected through the interhemispheric ipsilateral transcallosal approach. Postoperative MR imaging revealed complete resection of the tumor without complications (Fig. 3). 


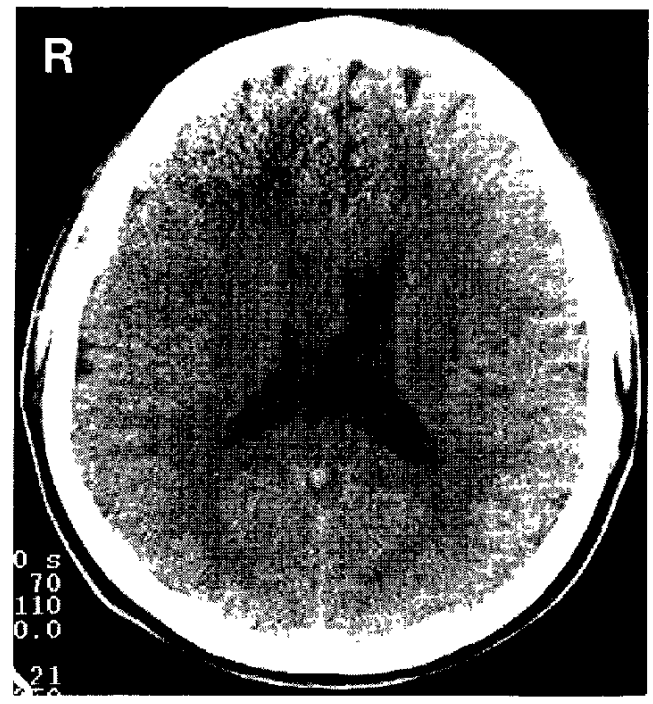

Macroscopically, this elastic and whitish tumor contained many vacuoles. No hemorrhage or necrosis was identified. Histological examination found the tumor consisted of both fine eosinophilic fibrillar matrix and aggregated cells with a high nuclear/ cytoplasmic ratio. There were many microcysts between the cells. Few astrocytic reactions were observed (Fig. 4).

Since the biological characteristics of this subepen-

Fig. 1 Preoperative computed tomography scan showing the subependymoma as an isodense tumor in the right lateral ventricle. No calcification is observed.

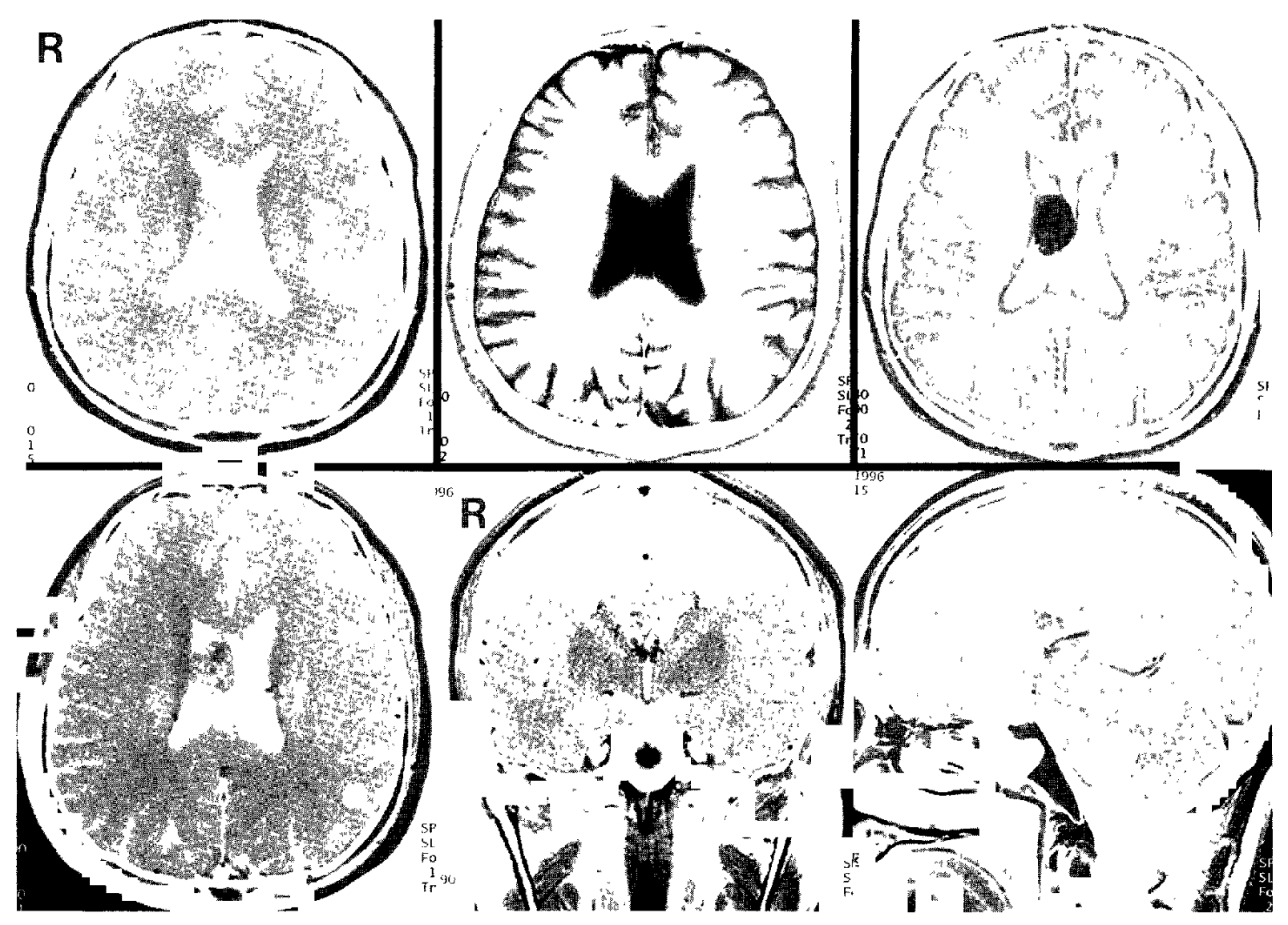

Fig. 2 Preoperative magnetic resonance images showing the subependymoma located in the body of right lateral ventricle as isointense on the $T_{1}$-weighted image (upper left) and hyperintense on the $\mathbf{T}_{2}$-weighted (upper center) and proton (upper right) images. There are microcysts inside the tumor. No intraparenchymal extension is identified. Partial enhancement was observed after gadolinium-diethylenetriaminepenta-acetic acid administration (lower row). 

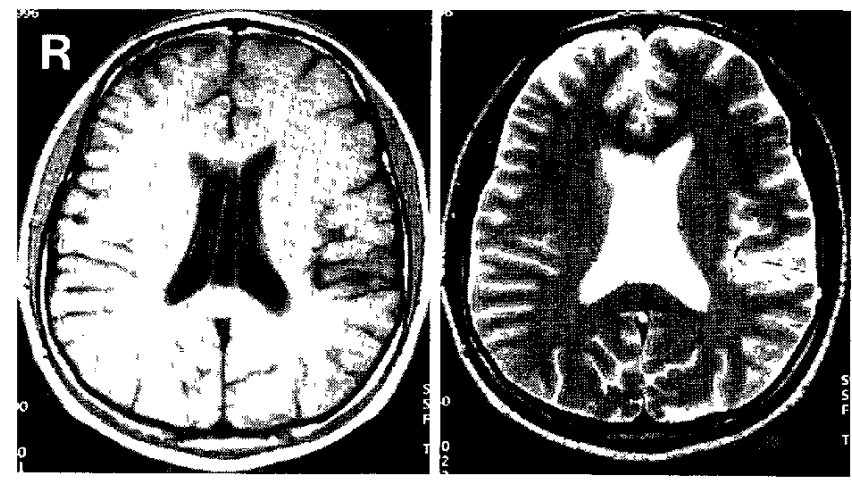

Fig. 3 Postoperative magnetic resonance image after total resection of the tumor as shown on $\mathrm{T}_{1}$-weighted (left) and $\mathrm{T}_{2}$-weighted (right) images.

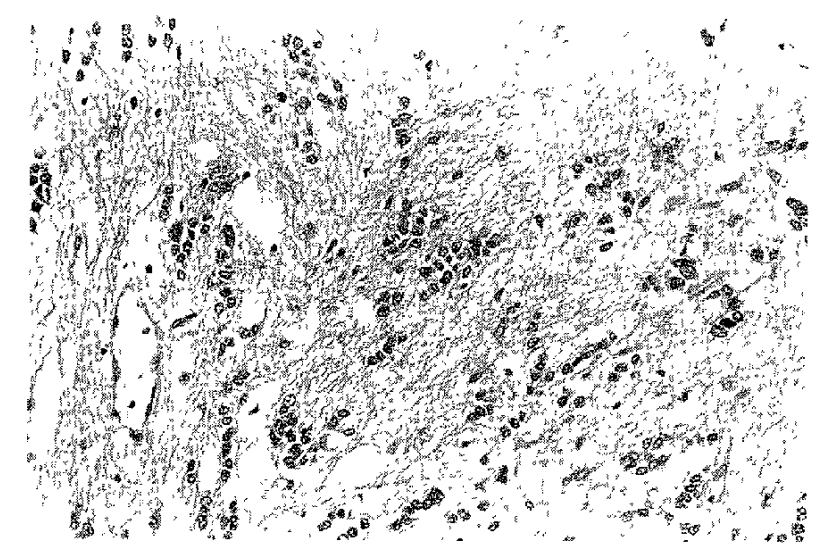

Fig. 4 Photomicrograph of the tumor specimen showing fine eosinophilic fibrillar matrix and aggregated cells with a high nuclear/ cytoplasmic ratio. Microcysts are also observed. No hemorrhage or necrosis is present.

dymoma were benign and complete resection was achieved, no adjunctive therapy was administered. The patient was doing well without symptoms 6 months following the operation.

\section{Discussion}

Subependymoma has similar histological characteristics to glioma and ependymoma, but is not classified with either of these tumors. The exact origin of subependymoma is still controversial. ${ }^{11)}$ Subependymoma has benign properties and a good prognosis, so adjunctive treatment is not required if the histological diagnosis is established.

The CT and MR imaging characteristics of in- traventricular subependymomas have been described previously. ${ }^{2,6,11,13)}$ MR imaging shows subependymoma as iso- or hypointense on the $\mathrm{T}_{1-}$ weighted image and hyperintense on the $T_{2}$-weighted and proton images. Cystic components are sometimes observed. ${ }^{13)}$ No or slight enhancement is caused by Gd-DTPA administration. Intraventricular tumors that should be differentiated from subependymoma include choroid plexus papilloma, meningioma, central neurocytoma, subependymal giant cell astrocytoma, germ cell tumor, and metastatic tumors. These intraventricular tumors are likely to be more enhanced with Gd-DTPA than subependymomas. ${ }^{2)}$ However, some subependymomas have been enhanced by Gd-DTPA. Therefore, CT and MR imaging cannot completely differentiate these intraventricular tumors. ${ }^{14]}$

About $40 \%$ of subependymomas are symptomatic. ${ }^{\text {b) }}$ Symptomatic subependymomas are $4 \mathrm{~cm}$ or more in diameter and are usually larger than "asymptomatic" subependymomas. ${ }^{11)}$ The subependymoma in our patient was relatively small compared to previous asymptomatic cases. Most ependymomas have localized attachments to the ventricular wall. However, subarachnoid space extension and/or secondary attachment to another ventricular wall may occur with growth, ${ }^{1,11)}$ and may cause complications during surgical extirpation. Asymptomatic subependymomas may become symptomatic due to subarachnoid hemorrhage or intratumoral hemorrhage. ${ }^{5,7)}$ Subependymomas can be completely resected, if small and/or not adherent to the ventricular wall. Many operative approaches, such as the transcortical, ipsilateral transcallosal, and contralateral transcallosal approaches, can be employed to treat intraventricular tumors. ${ }^{4,9,12)}$ We used the ipsilateral transcallosal approach in our case, due to the relatively medial location and absence of intraparenchymal extension.

Treatment protocols are substantially different for subependymoma and aggressive tumors. Radiation therapy is usually administered to aggressive tumors following surgery. In contrast, subependymomas do not require adjuvant therapy if they are totally extirpated. However, intraventricular tumors cannot be completely distinguished by neuroimaging, so histological confirmation following surgical extirpation is recommended. This relatively rare and benign lesion should be kept in mind as part of the differential diagnosis of intraventricular tumors.

The treatment protocol for asymptomatic intraventricular tumors is controversial. The subependymoma in our patient was incidentally found during a medical checkup. Increased public awareness of individual health concerns has resulted in widespread 
use of the medical checkup system in recent years. This system is expected to find a higher incidence of otherwise non-identified asymptomatic lesions. A lesion not found during medical checkup may become larger and symptomatic. Surgery is one of the treatment options to prevent symptoms. Intraventricular tumors generally tend to be larger than intraparenchymal ones, although they are asymptomatic. Surgical resection may become more difficult once the tumor becomes symptomatic due to secondary attachment to the ventricular wall. Informed consent should be obtained for surgical treatment of these asymptomatic tumors.

\section{References}

1) Azzarelli B, Rakate HL, Roessmann U: Subependymoma: a case report with ultrastructural study. Acta Neuropathol (Berl) 40: 279-282, 1977

2) Jelinek J, Smirniotopoulos JG, Parisi JE, Kanzer M: Lateral ventricular neoplasms of the brain: Differential diagnosis based of clinical, CT, and MR findings. AJR Am J Roentgenol 155: 365-372, 1990

3) Kim DG, Han MH, Chi JG, Cho KJ, Kim JH, Choi KS, Han DH: MRI of intracranial subependymoma: report of a case. Neuroradiology 35: 185-186, 1993

4) Lawton MT, Goffinos JG, Spetzler RF: The contralateral transcallosal approach: Experience with 32 patients. Neurosurgery 39: 729-735, 1996

5) Lindboe CF, Stolt-Nielsen A, Dale LG: Hemorrhage in highly vascularized subependymoma of the septum pellucidum: Case report. Neurosurgery 31: 741745,1992

6) Lobato RD, Sarabia M, Castro S, Esparza J, Cordobes F, Portillo JM, Rivas JJ: Symptomatic subependymo- ma: Report of four new cases studied with computed tomography and review of the literature. Neurosurgery 19: 594-598, 1986

7) Marra A, Dario A, Scamoni C, Cerati M, Crivelli G, Dorizzi A: Intraventricular subependymoma presenting as subarachnoid hemorrhage. Case report. J Neurosurg Sci 35: 213-215, 1991

8) Matsumura A, Ahyai A, Hori A, Schaake T: Intracerebral subependymoma. Clinical and neuropathological analyses with special reference to the possible existence of a less benign variant. Acta Neurochir (Wien) 96: 15-25, 1989

9) Rhoton AL, Yamamoto I, Peace DA: Microsurgery of the third ventricle: Part 2 -Operative approaches. Neurosurgery 8: 357-373, 1981

10) Scheinker IM: Subependymoma: a newly recognized tumor of subependymal derivation. J Neurosurg 2: 232-240, 1945

11) Scheithauer BW: Symptomatic subependymoma. J Neurosurg 49: 689-696, 1978

12) Shucart WA, Stein BM: Transcallosal approach to the anterior ventricular system. Neurosurgery 3: 339343,1978

13) Silverstein JE, Lenchik L, Stanciu MG, Shimkin PM: MRI of intracranial subependymomas. J Comput Assist Tomogr 19: 264-267, 1995

14) Yamasaki $T$, Kikuchi $H$, Higashi $T$, Yamabe $H$, Moritake K: Two surgically cured cases of subependymoma with emphasis on magnetic resonance imaging. Surg Neurol 33: 329-335, 1990

Address reprint requests to: S. Katayama, M.D., Department of Neurosurgery, Nishi-Kobe Medical Center, 5-7-1 Koujidai, Nishi-ku, Kobe 651-2273, Japan. 